\title{
Combination therapy with butyrate and docosahexaenoic acid for keloid fibrogenesis: an in vitro study*
}

\author{
Kazuhiro Torii ${ }^{1}$ \\ Michiko Aoyama-Ishikawa ${ }^{1}$ \\ Hiroto Terashi ${ }^{3}$
}

\author{
Noriaki Maeshige ${ }^{1,2}$ \\ Makoto Miyoshi ${ }^{1}$ \\ Makoto Usami ${ }^{1,4}$
}

DOI: http:/ / dx.doi.org/10.1590/abd1806-4841.20176198

\begin{abstract}
BACKGROUND: A single, effective therapeutic regimen for keloids has not been established yet, and the development of novel therapeutic approaches is expected. Butyrate, a short-chain fatty acid, and docosahexaenoic acid (DHA), a $\omega-3$ polyunsaturated fatty acid, play multiple anti-inflammatory and anticancer roles via their respective mechanisms of action.

ОвјестіVE: In this study, we evaluated the antifibrogenic effects of their single and combined use on keloid fibroblasts.

MetHods: Keloid fibroblasts were treated with butyrate (0-16 mM) and/or DHA (0-100 $\mu \mathrm{M})$ for 48 or $96 \mathrm{~h}$.

RESULTS: Butyrate inhibited cell proliferation, and $\alpha$-smooth muscle actin ( $\alpha$-SMA) and type III collagen expressions, with inhibition of the transforming growth factor (TGF)- $\beta 1$ and TGF- $\beta$ type I receptor expressions and increased prostaglandin E2 with upregulation of cyclooxygenase-1 expression with induction of histone acetylation. DHA inhibited $\alpha$-SMA, type III collagen, and TGF- $\beta$ type I receptor expressions. Then, the butyrate/DHA combination augmented the antifibrogenic effects, resulting in additional inhibition of $\alpha$-SMA, type I and III collagen expressions, with strong disruption of stress fiber and apoptosis induction. Moreover, the butyrate/DHA combination inhibited the cyclooxygenase-2 expression, suggesting stronger antiinflammatory effect than each monotherapy.

STUDY LIMITATIONS: Activation in keloid tissue is affected not only by fibroblasts but also by epithelial cells and immune cells. Evaluation of the effects by butyrate and DHA in these cells or in an in vivo study is required.

CONCLUSION: This study demonstrated that butyrate and docosahexaenoic acid have antifibrogenic effects on keloid fibroblasts and that these may exert therapeutic effects for keloid.
\end{abstract}

Keywords: Butyrates; Docosahexaenoic acids; Fibroblasts; Fibrosis; Keloid; Prostaglandins E

\section{INTRODUCTION}

Keloid and hypertrophic scars are dermal fibroproliferative disorders unique to humans, causing cosmetic deformities and psychological stress, which consequently impair the patient's quality of life. ${ }^{1}$ Current treatment modalities such as surgery, radiation, and immunomodulation have been demonstrated to have therapeutic effects, but no modality has completely eliminated the risk of recurrence., ${ }^{2,3}$

Keloid formation has been linked to aberrant fibroblast activity resulting in increased expression of many potent cytokines and growth factors, especially transforming growth factor (TGF)- $\beta 1$. ${ }^{4}$ The binding of TGF- $\beta 1$ to TGF- $\beta$ receptors, including TGF- $\beta$ type I receptor (TGF- $\beta$ RI) and TGF- $\beta$ type II receptor (TGF- $\beta$ RII), leads to activation of the TGF- $\beta$ RI by TGF- $\beta$ RII-mediated phosphorylation. ${ }^{5}$ As a result of the response to TGF- $\beta 1$, cellular recruitment of the fibroblasts is enhanced, resulting in fibroblast differentiation into myofibroblasts; the differentiation causes intense cell proliferation, apoptosis resistance, and synthesis of extracellular matrix proteins, especially type I collagen (collagen I) and type III collagen (collagen III). ${ }^{6}$ In fibroproliferative tissues, fibroblast differentiation into myofibroblasts is commonly identified by $\alpha$-smooth muscle actin ( $\alpha$-SMA) expression, which is altered by stress fiber formation in the downstream signaling of TGF- $\beta 1 .{ }^{7}$ Functionally, these cells can generate contractile force and promote fibrosis. Therefore, the suppression of these parameters may be useful in therapeutic approaches for keloids. In addition, keloid fibroblasts (KFBs) secrete less prostaglandin $\mathrm{E}_{2}\left(\mathrm{PGE}_{2}\right)$ than normal dermal fibroblasts (NFBs), ${ }^{8}$ and $\mathrm{PGE}_{2}$ has been reported to possess antifibrogenic activities such as inhibition of fibroblast proliferation, migration, and collagen expression in KFBs. ${ }^{8,9}$

Short-chain fatty acids (FAs) are the endproducts of anaerobic bacterial fermentation of dietary fibers in the colon. These FAs, predominantly butyrate and propionate, have a histone deacetylase (HDAC) inhibitor activity and play multiple roles such as apoptosis induction, proliferation regulation, and differentiation in the colonic epithelium. ${ }^{10,11}$ As for the effects of short-chain FAs on $\mathrm{PGE}_{2}$ secretion, we have reported that butyrate and trichostatin A, a typical HDAC inhibitor, indicates more $\mathrm{PGE}_{2}$ secretion and stronger atten-

\footnotetext{
Received on 14.04.2016.

Approved by the Advisory Board and accepted for publication on 02.08.2016.

* Study conducted at the Graduate School of Health Sciences, Kobe University - Kobe, Japan.

Financial support: This work was supported by JSPS KAKENHI Grant Number 23592648.

Conflict of interest: None.

Division of Nutrition and Metabolism, Department of Biophysics, Graduate School of Health Sciences, Kobe University - Kobe, Japan.

Department of Rehabilitation Science, Graduate School of Health Sciences, Kobe University - Kobe, Japan.

Department of Plastic Surgery, Graduate School of Medicine, Kobe University - Kobe, Japan.

Department of Nutrition, Kobe University Hospital - Kobe, Japan. 
uation of nuclear factor- $\mathrm{B}$ activation than propionate in lipopolysaccharide-activated human peripheral blood mononuclear cells. ${ }^{12}$ Butyrate or other HDAC inhibitors have been reported to have antifibrogenic effects on several mesenchymal cells, including human lung fibroblasts, showing inhibition of cell proliferation, collagen production, and $\alpha$-SMA expression through histone acetylation. ${ }^{13-15}$ In our study using NFBs, butyrate exerted stronger antifibrogenic effects than propionate. ${ }^{16}$ Moreover, butyrate was reported to be a more potent HDAC inhibitor than propionate. ${ }^{10}$ However, these effects of butyrate on KFBs have not been investigated.

Docosahexaenoic acid (DHA) and eicosapentaenoic acid are major FAs in $\omega-3$ polyunsaturated FAs of marine organisms, including fish oil. These FAs have anti-inflammatory and anticancer effects. ${ }^{17,18}$ Antifibrogenic effects of DHA have been reported in human peritoneal fibroblasts, including inhibition of TGF- $\beta 1$, vascular endothelial growth factor, and collagen I expressions. ${ }^{19}$ In our study using NFBs, DHA exerted stronger antifibrogenic effects than eicosapentaenoic acid, indicating inhibition of $\alpha$-SMA, TGF- $\beta 1$, and collagen III expressions, in concurrence with a report on human prostate carcinoma cell line. ${ }^{16,20}$ DHA is a ligand of peroxisome proliferator-activated receptor $\gamma(\operatorname{PPAR} \gamma)$, and lipid mediators derived from DHA are potent agonists of PPAR $\gamma .{ }^{21}$ Many studies reported that PPAR $\gamma$ expression or activation inhibited collagen I, $\alpha$-SMA, and TGF- $\beta$ RI expressions in mouse skin and embryonic and human dermal fibroblasts. ${ }^{21,22}$ Therefore, PPAR $\gamma$ expression regulation by DHA might be a possible underlying mechanism of antifibrogenic effects. However, these effects of DHA on KFBs have not been investigated.

Although we have reported that butyrate and DHA showed inhibition of profibrotic factors in NFBs, the effect of these FAs in KFBs remain unclear. In the present study, we investigated the antifibrogenic effects of single and combined treatment with butyrate and DHA on KFBs by measuring profibrotic factors, cell proliferation, apoptosis, and stress fiber formation, and the underlying mechanism of the antifibrogenic effects on each FA by measuring $\mathrm{PGE}_{2}$ secretion, histone acetylation, and PPAR $\gamma$ expression level.

\section{METHODS}

Primary keloid fibroblast cultures

KFBs were obtained from chest and earlobe keloid tissues of two Japanese patients. The protocol for tissue collection was approved by the ethics review board at Kobe University Graduate School. Five or 6 pieces of the minced and deepithelialized samples were placed on a 100-mm tissue culture dish (Iwaki, Tokyo, Japan) and immersed in 2 mL Dulbecco's modified Eagle medium (DMEM; Wako Pure Chemical Industries, Osaka, Japan) supplemented with $10 \%$ fetal bovine serum (FBS; Nichirei, Tokyo, Japan), penicillin (50 $\mathrm{U} / \mathrm{mL})$, and streptomycin $(50 \mu \mathrm{g} / \mathrm{mL}$; MP Biomedicals, Illkirch, France). On the following day, $15 \mathrm{~mL}$ of the medium was added to each dish. The culture medium was changed every 2 or 3 days until approximately $80 \%$ confluence was reached. The cells were passaged by incubation at $37^{\circ} \mathrm{C}$ with $0.05 \%$ trypsin and $0.02 \%$ EDTA, and plated in culture dishes.

\section{Cell culture}

KFBs were grown at a $37^{\circ} \mathrm{C} \mathrm{CO}_{2}$ incubator, using DMEM with $10 \%$ FBS. Only cells from passage 3 to 6 were used in this ex- periment. Trypan blue staining was performed to distinguish live cells from dead cells and absolute cell counts. For experimentation, KFBs were seeded into 6-well flat bottom plates (Iwaki) at a concentration of $2.8 \times 10^{5}$ cells/well and 96-well flat bottom plates (Iwaki) at a concentration of $8.0 \times 10^{3}$ cells/well.

\section{FA treatment}

Sodium butyrate (Sigma, St. Louis, MO) and sodium DHA (Sigma) were used. To evaluate the effects of the monotherapy, butyrate or DHA was applied at 0,4 , and $16 \mathrm{mM}$ or 0 and $100 \mu \mathrm{M}$, respectively, after our experiments on NFBs. ${ }^{16}$ To evaluate the combination treatment, butyrate was applied at 4 and $16 \mathrm{mM}$ with DHA at $100 \mu \mathrm{M}$. BrdU assays

KFBs were seeded into 96-well flat bottom plates and cultured in culture medium with $10 \%$ FBS. After $24 \mathrm{~h}$, FAs were added and the cells were incubated for $48 \mathrm{~h}$, followed by analysis using BrdU incorporation assay (Roche, Basel, Switzerland), according to the manufacturer's instructions.

RNA extraction, cDNA synthesis, and quantitative real-time polymerase chain reaction analysis

For the experiments, KFBs were seeded into each well of 6-well flat bottom plates and cultured in culture medium with $10 \%$ FBS. After $24 \mathrm{~h}$, FAs were added and the cells were incubated for $48 \mathrm{~h}$. Then, the cells were processed for total RNA isolation using TRIzol (Invitrogen, Carlsbad, CA) according to the manufacturer's instructions and reverse transcribed to yield a single-stranded cDNA, using iScript cDNA synthesis kits (Bio-Rad, Hercules, CA) according to the manufacturer's instructions. The cDNAs were used for the subsequent quantitative real-time polymerase chain reaction (PCR) analysis, using SYBR Premix Ex Taq II (Takara Bio, Otsu, Japan) with each primer (Table 1). ${ }^{16,23-26}$ The PCR reactions were run on iCycler IQ (Bio-Rad, Hercules, CA) for 40 cycles at $95^{\circ} \mathrm{C}$ for $30 \mathrm{~s}$, at an annealing temperature (Table 1) for $30 \mathrm{~s}$, and at $72.0^{\circ} \mathrm{C}$ for $30 \mathrm{~s}$. Post-polymerase chain reaction melting curves were confirmed by the specificities of single-target amplification, and the relative expressions of each gene were calculated in duplicate based on glyceraldehyde-3-phosphate dehydrogenase (GAPDH) expression.

Western blotting

KFBs were seeded into each well of 6-well flat bottom plates and cultured in culture medium with $10 \%$ FBS. After $24 \mathrm{~h}$, FAs were added and the plates were incubated for $48 \mathrm{~h}$. After preparation of the KFBs in $1.5 \mathrm{~mL}$ tubes, they were suspended to $100 \mu \mathrm{L}$ of ProPrep (iNtRON, Gyeonggi-do, Korea), according to the manufacturer's instructions. Five microliters of the cell supernatants was used to measure the protein concentration, using Lowry's method (RC DC Protein Assay Kit, Bio-Rad, Hercules, CA). Western blotting was performed as described previously, using primary antibodies against $\alpha$-SMA (1:400; Sigma), acetyl-histone H3 (1:1000; Cell Signaling Technology Inc., Danvers, CO), PPAR $\gamma$ (1:800; Santa Cruz Biotechnology Inc., Santa Cruz, CA), GAPDH (1:40000; Sigma), and appropriate horseradish peroxidase-conjugated secondary antibody. ${ }^{27}$ Densitometric results were analyzed using the Image J software (National Institutes of Health, Bethesda, MD).

Measurement of $P G E_{2}$ levels in culture supernatants

$\mathrm{PGE}_{2}$ concentrations were measured using enzyme immunoassay kits (R\&D Systems, Minneapolis, MN), according to the 
manufacturer's protocol, and an enzyme-linked immunosorbent assay reader (Benchmark Microplate Reader, Bio-Rad, Tokyo, Japan).

Immunofluorescence staining

The morphological features of F-actin and the nuclei in KFBs were analyzed by immunofluorescence staining, as described previously, using phallotoxins (1:40; Invitrogen, Carlsbad, CA) and 4',6-diamidino-2-phenylindole (1:1000; Dojin, Kumamoto, Japan). ${ }^{16}$ Immunofluorescent staining patterns were observed with a BX50 fluorescence microscope at $\times 200$ magnification (Olympus, Tokyo, Japan) and recorded with a digital camera (EOS Kiss X4, Canon, Tokyo, Japan). Apoptotic cells were identified by nucleus condensation and fragmentation, and nuclei from 10 random fields of each coverslip were examined at $\times 100$ magnification.

\section{Statistical analysis}

Data from independent experiments were used to calculate the mean \pm SD values. Differences were considered significant if $p<$ 0.05 , as determined by the Tukey-Kramer post hoc test.

\section{RESULTS}

Profibrotic factor and TGF- $\beta 1$ signal expressions

We evaluated the effects of butyrate and DHA on profibrotic factors in KFBs from chest. As for $\alpha$-SMA expression, butyrate or DHA inhibited $\alpha$-SMA mRNA expression similarly $(p<0.01$; Figure 1). The butyrate/DHA combination augmented the inhibitory effect of butyrate $(13.1 \%$ of the control; $p<0.01)$. This change in mRNA level was obvious in the protein level. As for collagen expression, the butyrate or DHA treatment inhibited collagen III expression $(14.2 \%$ or $68.2 \%$, respectively; $p<0.01$ ). The butyrate/DHA combination increased the inhibitory effect, resulting in additional inhibition of collagen III (7.5\% of the control) and collagen I expressions $(49.6 \%$ of the control; $p<0.05)$. Since butyrate and DHA exerted the similar effects on these profibrotic factors in KFBs from earlobe, the following experiments were conducted in KFBs from chest.

To further characterize the inhibition of profibrotic factors, we investigated the TGF- $\beta 1$ signal mRNA expression (Figure 1). Butyrate

\section{TABLE 1: Primers used for real-time polymerase chain reaction}

\begin{tabular}{llll} 
Gene & Forward $\left(\mathbf{5}^{\prime} \mathbf{-} \mathbf{3}^{\prime}\right)$ & Reverse $\mathbf{( 5}^{\prime} \mathbf{-} \mathbf{3}^{\prime} \mathbf{)}$ & Annealing \\
\hline GAPDH & CATCAAGAAGGTGGTGAAGC & CCTCCCCAGCAAGAATGTCT & $62.5^{\circ} \mathrm{C}$ \\
$\alpha$-SMA & CGTGGGTGACGAAGCACAG & GGTGGGATGCTCTTCAGGG & $62.5^{\circ} \mathrm{C}$ \\
TGF- $\beta 1$ & GGGACTATCCACCTGCAAGA & CCTCCTTGGCGTAGTAGTCG & $62.5^{\circ} \mathrm{C}$ \\
collagen I & GTGCTAAAGGTGCCAATGGT & ACCAGGTTCACCGCTGTTAC & $57.5^{\circ} \mathrm{C}$ \\
collagen III & TATCGAACACGCAAGGCTGTGAGA & GGCCAACGTCCACACCAAATCTT & $65.8^{\circ} \mathrm{C}$ \\
COX-1 & ATGATGGGCCTGCTGTGGA & CCAACACTCACCATGCCAAAC & $61.5^{\circ} \mathrm{C}$ \\
COX-2 & TCCCTGAGCATCTACGGTTTG & AACTGCTCATCACCCCATTCA & $61.7^{\circ} \mathrm{C}$ \\
TGF- $\beta$ RI & GGTCTTGCCCATCTTCACAT & TCTGTGGCTGAATCATGTCT & $56.0^{\circ} \mathrm{C}$ \\
TGF- $\beta$ RII & TTTGGGCTTTCCCTGCGTCT & TCTGGAGCCATGTATCTTGCAGTTC & $64.5^{\circ} \mathrm{C}$
\end{tabular}

A

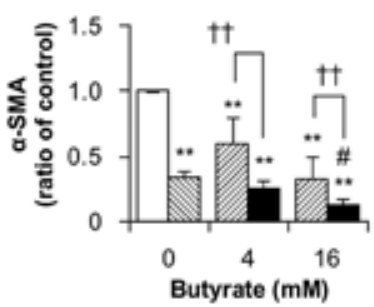

B
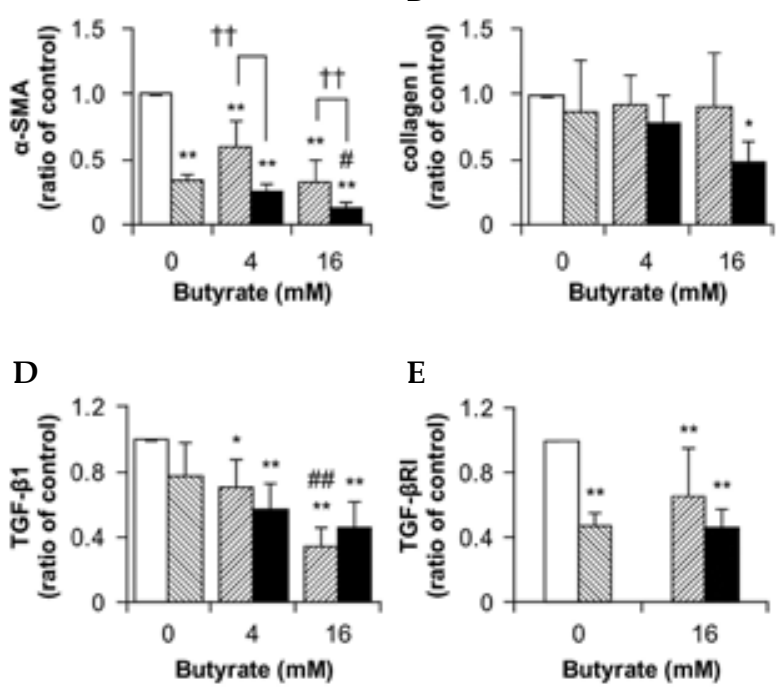

E

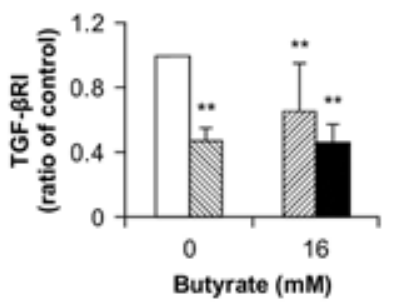

C

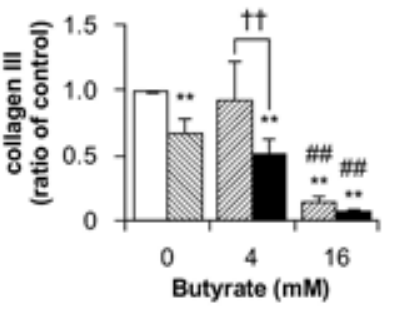

G

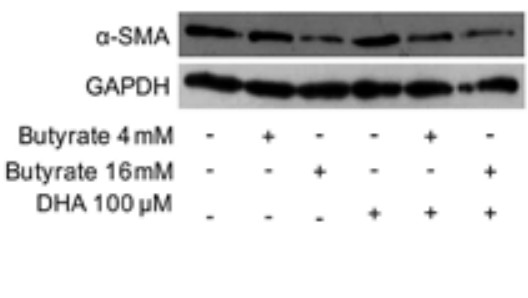

F

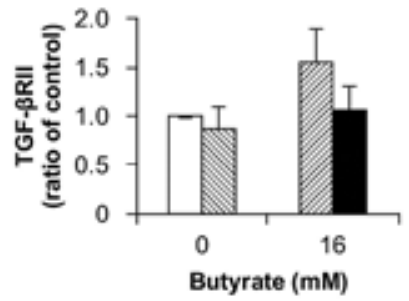

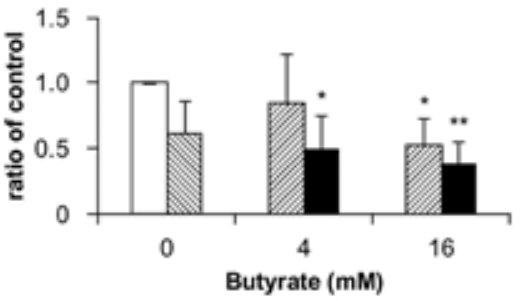

Control DHA Q Butyrate

Butyrate + DHA

KFBs were exposed for $48 \mathrm{~h}$ to the indicated concentrations of butyrate with/without $100 \mu$ M DHA. The (a) $\alpha$-SMA, (b) collagen I, (c) collagen III, (d) TGF- $\beta 1$, (e) TGF- $\beta$ RI, and (f) TGF- $\beta$ RII mRNA expressions were analyzed by real-time polymerase chain reaction. $(\mathrm{g})$ The $\alpha$-SMA and GAPDH protein expressions were analyzed by western blotting. Results from a representative experiment are shown. Similar results were obtained from 6 independent experiments. The graph shows the $\alpha$-SMA/GAPDH ratio. Data from 6 independent experiments were used to calculate mean $\pm \mathrm{SD}\left({ }^{*} \mathrm{p}<0.05\right.$ and ${ }^{* *} \mathrm{p}<0.01$, vs. the control; $\# \mathrm{p}<0.05$ and $\# \# \mathrm{p}<0.01$, vs. DHA; $\mathrm{tp}<0.05$ and $+\dagger \mathrm{p}<0.01$, butyrate vs. butyrate + DHA)

FIGURE 1: Effects of butyrate with/without DHA on the profibrotic factors and TGF-ß1 signaling expressions in KFBs 
inhibited the TGF- $\beta 1$ ( $70.4 \%$ of the control and $34.1 \%$ at 4 and $16 \mathrm{mM})$ and TGF- $\beta R I$ expressions $(65.8 \%$ at $16 \mathrm{mM} ; p<0.01)$. DHA inhibited TGF- $\beta R I$ expression $(47.2 \% ; p<0.01)$. However, butyrate or DHA did not affect the TGF- $\beta$ RII expression. The effect of the butyrate/DHA combination was similar to that of the monotherapy in each FA.

\section{KFB growth, survival, and apoptosis}

Butyrate or the butyrate/DHA combination reduced the number of cells in the control to $51.2 \%$ or $48.8 \%$ with $4 \mathrm{mM}$ butyrate and $40.8 \%$ or $43.2 \%$ with $16 \mathrm{mM}$ butyrate $(p<0.01)$, respectively, with no change in cell viability (Figure 2). These changes in cell number were accompanied by those obtained in the 5-bromo- 2 -deoxyuridine (BrdU) incorporation assay results, that is, $23.3 \%$ or $23.6 \%$ in 4 $\mathrm{mM}$ butyrate and $9.4 \%$ or $10.6 \%$ in $16 \mathrm{mM}$ butyrate, respectively ( $p$ $<0.01)$. DHA indicated no effect.

DNA fragmentation was clearly observed after $96 \mathrm{~h}$ of treatment with $16 \mathrm{mM}$ butyrate $(p<0.01)$ but was not detected after 48 $\mathrm{h}$ of treatment (data not shown), accompanied with high cellular viability. The butyrate/DHA combination-induced DNA fragmentation slightly augmented the effect of butyrate.

\section{in KFBs}

Alteration of histone acetylation and PPAR $\gamma$ expression

Butyrate induced histone $\mathrm{H} 3$ acetylation dose-dependently by 3.7- and 5.5-fold at 4 and $16 \mathrm{mM}$, respectively $(p<0.01)$, indicating inhibition of HDAC activity. In contrast, DHA did not alter this activity (Figure 3). The result of the butyrate/DHA combination was similar to that of the butyrate-only treatment.
To determine the mechanism of action of DHA, we also evaluated the PPAR $\gamma$ expression (Figure 3). Butyrate or DHA did not induce a significant PPAR $\gamma$ expression at $48 \mathrm{~h}$ of treatment.

F-Actin arrangement

Butyrate at $16 \mathrm{mM}$ or DHA at $100 \mu \mathrm{M}$ decreased stress fiber formation, with butyrate having a stronger effect than DHA (Figure 4). The butyrate/DHA combination also strongly disrupted the stress fibers in the cytoplasm after $48 \mathrm{~h}$ of treatment.

\section{$P G E_{2}$ synthesis}

Butyrate increased $\mathrm{PGE}_{2}$ secretion by 4.6 -fold of the control at $16 \mathrm{mM}$ ( $p<0.01$; Figure 5), whereas DHA did not. The result of the butyrate/DHA combination was similar to that of the butyrate-only treatment.

Butyrate significantly increased the COX-1 expression level to $174.9 \%$ of the control $(p<0.05)$. Butyrate or DHA slightly suppressed the COX-2 expression to $70.2 \%$ or $82.6 \%$, respectively, but not significantly. The butyrate/DHA combination suppressed the COX-2 expression to $61.9 \%(p<0.05)$.

\section{DISCUSSION}

This is the first report to indicate the antifibrogenic effects of butyrate and DHA on KFBs. This study presents 3 novel findings. First, butyrate inhibited cell proliferation and $\alpha$-SMA, collagen III, TGF- $\beta 1$, and TGF- $\beta$ RI expressions and induced apoptosis, $\mathrm{PGE}_{2}$ secretion, COX-1 expression, stress fiber disruption, and histone acetylation. Second, DHA inhibited $\alpha$-SMA, collagen III, and
A

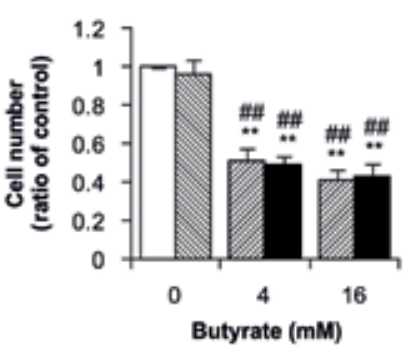

B

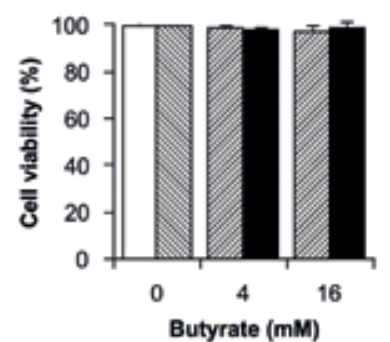

C

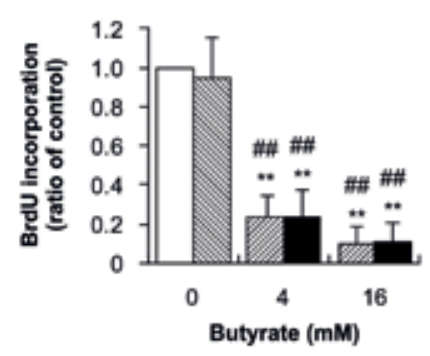

E

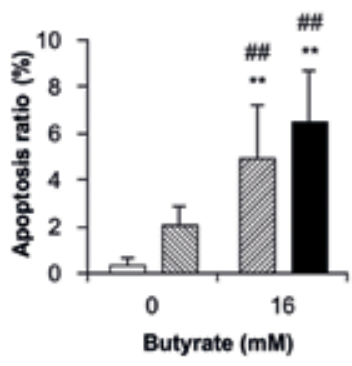

Control DHA Butyrate $\square$ Butyrate + DHA

D
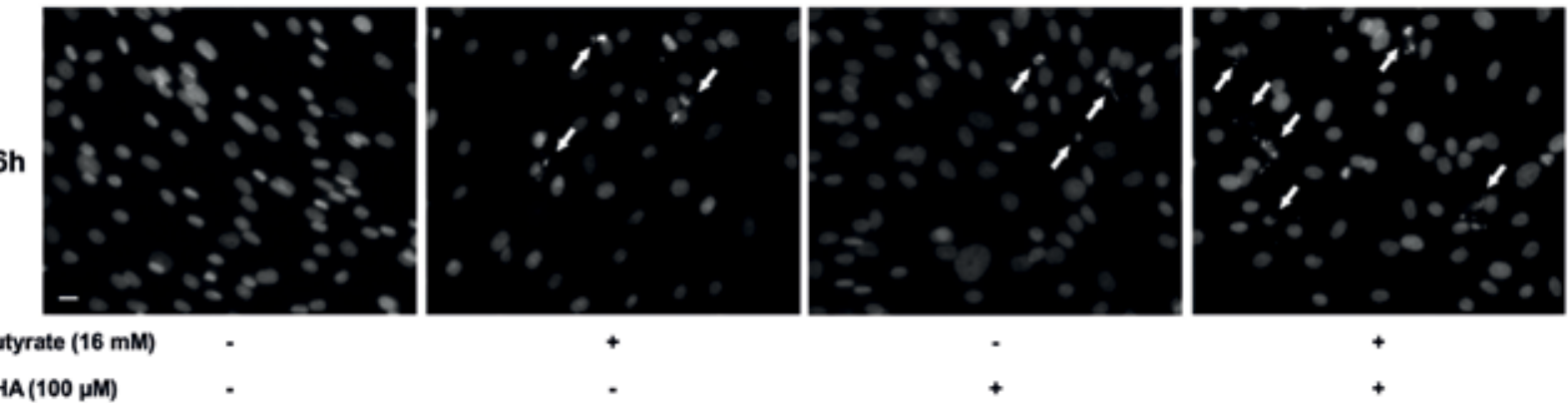

Butyrate (16 mM) DHA (100 $\mu \mathrm{M})$

KFBs were exposed for $48 \mathrm{~h}$ to the indicated butyrate concentrations with/without $100 \mu \mathrm{M}$ DHA. (a) The cell number and (b) viability were determined by trypan blue staining. (c) Proliferation was assessed using the BrdU incorporation assay. Data from 6 (cell number and viability) and 10 (BrdU assay) separate cultures were used to calculate mean \pm SD. (d) White arrows indicate DNA fragmentation. KFBs were exposed for $96 \mathrm{~h}$ to $16 \mathrm{mM}$ butyrate with/without $100 \mu \mathrm{M}$ DHA. The micrographs are representative of all the cell cultures. (e) DNA fragmentation from 10 random fields of each coverslip was used to calculate mean $\pm \mathrm{SD}\left({ }^{* *} \mathrm{p}<0.01\right.$, vs. the control cultures; \#\#p $<0.01$, vs. DHA). Scale bar, $20 \mu \mathrm{m}$

FIGURE 2: Effects of butyrate with/without DHA on KFB proliferation, survival, and apoptosis 
TGF- $\beta R I$ expressions but did not alter PPAR $\gamma$ expression. Third, the butyrate/DHA combination augmented the inhibitory effects on $\alpha$-SMA, collagen I, collagen III, TGF- $\beta 1$, and TGF- $\beta$ RI expressions; inhibited cell proliferation; induced apoptosis; and strongly dis-
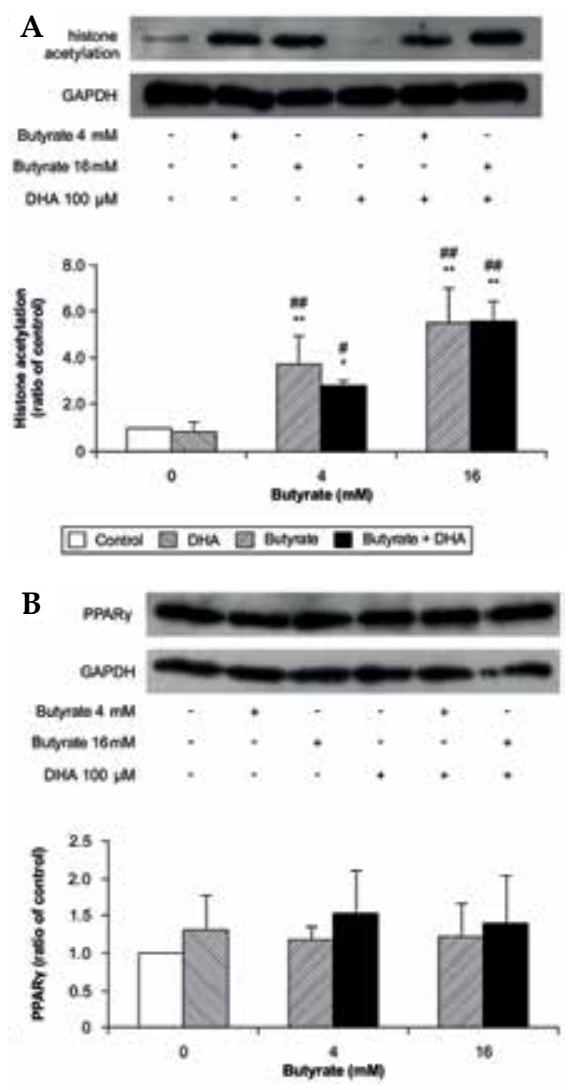

KFBs were exposed for $48 \mathrm{~h}$ to the indicated concentrations of butyrate with $100 \mu \mathrm{M}$ DHA. The protein expressions of (a) histone acetylation and (b) PPAR $\gamma$ were analyzed using western blotting. Results from a representative experiment are shown. Similar results were obtained from 6 independent experiments. The graphs show the histone acetylation/ GAPDH and PPAR $\gamma /$ GAPDH ratios. Data from 6 independent experiments were used to calculate the mean $\pm \mathrm{SD}$ values ( ${ }^{*} \mathrm{p}<0.05$ and ${ }^{* *} \mathrm{p}<0.01$, vs. the control)

FIGURE 3 : Effects of butyrate with/without DHA on histone acetylation and PPAR $\gamma$ expression in KFBs rupted stress fibers. These findings suggest the therapeutic effect of the butyrate/DHA combination on the fibrogenesis of keloids.

The present finding of strong inhibition of cell proliferation without cell viability change by butyrate is in agreement with that of our previous report using NFBs and other reports using several normal fibroblasts. ${ }^{16,28,29}$ This agreement suggests the efficacy of butyrate for not only normal fibroblasts but also activated fibroblasts in fibroproliferative disorders.

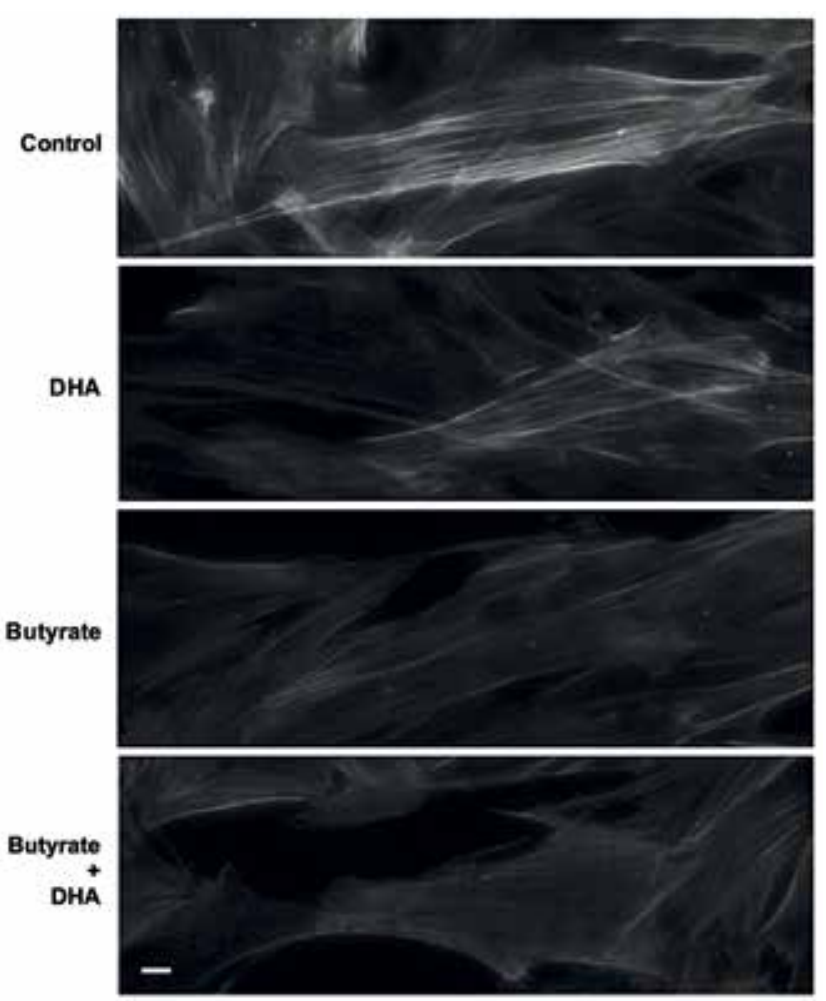

KFBs were exposed to DHA $(100 \mu \mathrm{M})$, butyrate $(16 \mathrm{mM})$, and butyrate with DHA (16 and $100 \mu \mathrm{M}$, respectively) for $48 \mathrm{~h}$. The morphological organizations of F-actin and the nuclei in KFBs were analyzed by immunofluorescence staining with phallotoxins (F-actin) and 4',6-diamidino-2-phenylindole (nuclei). The micrographs are representative of all the cell cultures. Scale bar, $10 \mu \mathrm{m}$

FIGURE 4 : Disruption of stress fibers by butyrate and DHA in KFBs

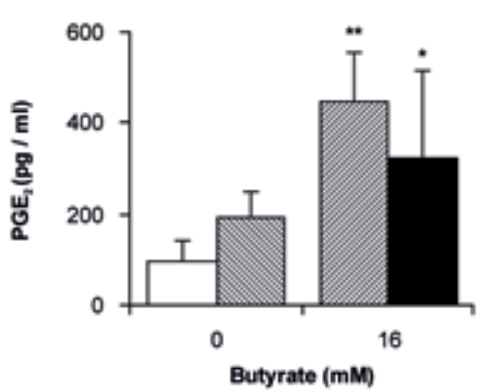

b

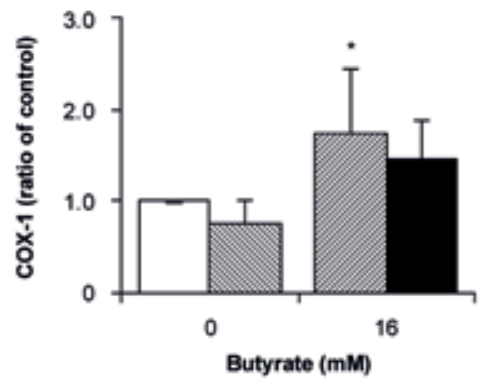

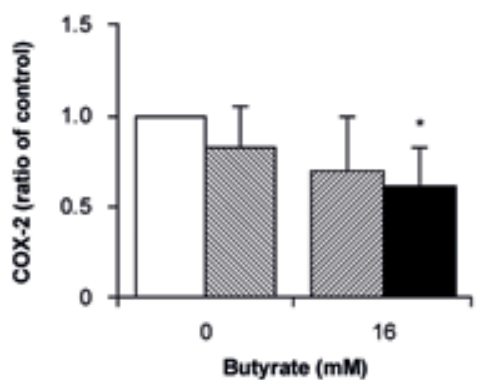

Control DHA Butyrate $\square$ Butyrate + DHA

KFBs were exposed for $48 \mathrm{~h}$ to the indicated concentrations of butyrate with $100 \mu \mathrm{M}$ DHA. (a) PGE2 concentrations in the medium were then measured. The mRNA expressions of (b) COX-1 and (c) COX-2 were analyzed by real-time polymerase chain reaction. Data from 6 independent experiments were used to calculate mean \pm SD ${ }^{*} \mathrm{p}<0.05$ and ${ }^{* *} \mathrm{p}<0.01$, vs. the control; $\# \mathrm{p}$ $<0.05$ and \#\#p $<0.01$, vs. DHA).

FIGURE 5 : Effects of butyrate with/without DHA on PGE2 secretion, and COX-1 and COX-2 mRNA expressions in KFBs 
The butyrate-induced histone acetylation is in agreement with a number of reports indicating antifibrogenic effects with the mechanism of cell cycle arrest at the $G_{1} / S$ phase and changes in a number of cell cycle regulatory gene expressions in rat embryonic fibroblasts. ${ }^{13,30,31}$ Considering our results and those of the other reports, we suggest that the mechanism of the antiproliferative activity in KFBs could be due to the butyrate-induced histone acetylation. Our result of DNA fragmentation after $96 \mathrm{~h}$ of treatment with butyrate coincides with the report using porcine fetal fibroblasts. ${ }^{29}$ The stronger induction of apoptosis by the butyrate/DHA combination than that by monotherapy is in agreement with reports indicating the mechanism of synergistic induction of apoptosis in colonocytes. ${ }^{32}$ From those reports, we suspect that the butyrate and DHA combination synergistically enhances apoptosis via an additional intrinsic mitochondrial pathway to a FAs-mediated extrinsic pathway reported to be activated in colonic cells by butyrate. ${ }^{33}$ Further studies to elucidate this mechanism in KFBs are required.

The inhibition of collagen III and $\alpha$-SMA expressions by butyrate or DHA is in agreement with our previous report on NFBs. ${ }^{16}$ The butyrate/DHA combination augmented the antifibrogenic effects, resulting in additional suppression of $\alpha$-SMA, collagen I, and collagen III expressions, which was stronger than that by butyrate monotherapy. Although 24-h FA treatment of KFBs did not show antifibrogenic effects in preliminary experiment different from those of NFBs, ${ }^{16} 48 \mathrm{~h}$ FA treatment showed strong antifibrogenic effects in KFBs, therefore suggesting that the long term FA treatment in fibroproliferative disorders requires cell activity inhibition.

In terms of TGF- $\beta 1$ signaling, the inhibition of TGF- $\beta 1$ and TGF- $\beta$ RI mRNA expressions by butyrate indicates that butyrate could inhibit TGF- $\beta 1$ signaling, followed by inhibitions in $\alpha$-SMA and collagen expressions. Meanwhile, stress fiber disruption by butyrate as well as histone acetylation in the present study is in agreement with the report on human lung cell line demonstrating stress fiber disruption by histone acetylation using the phenylbutyrate-based HDAC inhibitor. ${ }^{34}$ Furthermore, the inhibition of $\alpha$-SMA expression accompanied with stress fiber disruption by butyrate in the present study is in agreement with our previous report on NFBs, and stress fiber formation is reported to upregulate $\alpha$-SMA expression in fibroblasts. ${ }^{16,35}$ Therefore, stress fiber regulation would be one of the mechanisms in antifibrogenic effects by butyrate in addition to inhibition of TGF- $\beta 1$ signaling.

DHA induced neither histone acetylation nor drastic stress fiber disruption, as observed in the butyrate treatment in this study. Therefore, the mechanism of the antifibrogenic effects of DHA differs from that of butyrate. At first, we hypothesized that the anti- fibrogenic mechanism of DHA was via the upregulation of PPAR expression. However, in the present study, DHA did not alter the PPAR $\gamma$ expression at $48 \mathrm{~h}$ of treatment, in agreement with Ghosh's study using naturally occurring and synthetic pharmacological PPAR $\gamma$ ligands, showing no change in total cellular PPAR $\gamma$ level but an increase in PPAR $\gamma$ nuclear levels and activated PPAR $\gamma$ resulting in antifibrogenic effects. ${ }^{36}$ Therefore, it can be speculated that the antifibrogenic effects of DHA is due to the change in PPAR $\gamma$ nuclear levels or PPAR $\gamma$ activation. However, this requires confirmation in further experiments with KFBs.

The finding that $\mathrm{PGE}_{2}$ secretion and COX-1 expression increased with the butyrate treatment in KFBs is in agreement with that of our previous report on human peripheral blood mononuclear cells. ${ }^{12}$ Furthermore, Taniura et al. reported that trichostatin A, a potent $\mathrm{HDAC}$ inhibitor increased $\mathrm{PGE}_{2}$ secretion with upregulation of COX-1 expression in human astrocyte cells. ${ }^{37}$ Altogether, their results and our results indicate that the mechanism of increased $\mathrm{PGE}_{2}$ secretion in the present study could be due to increasing COX-1 expression by the butyrate-induced HDAC inhibitor activity. $\mathrm{PGE}_{2}$ was reported to inhibit collagen synthesis and $\alpha$-SMA in KFBs activated by TGF- $\beta 1$ and fibroblasts of idiopathic pulmonary fibrosis. ${ }^{38,39}$ Therefore, we suggest that the increase in $\mathrm{PGE}_{2}$ secretion by butyrate could be an underlying mechanism of profibrotic factor inhibition. Although the effect of the butyrate/DHA combination on $\mathrm{PGE}_{2}$ secretion was similar to that of the butyrate monotherapy, the butyrate/DHA combination inhibited COX-2 expression, suggesting the importance to control inflammatory signaling in keloid tissues. ${ }^{40}$ Considering this report, we suggest that an increase in $\mathrm{PGE}_{2}$ secretion with downregulation of COX-2 expression could be beneficial for keloid therapies because of the anti-inflammatory and antifibrogenic effects. However, further studies to elucidate the anti-inflammatory effects of the butyrate/DHA combination are needed.

Activation in keloid tissue is affected not only by fibroblasts but also by epithelial cells and immune cells such as keratinocytes and neutrophils. ${ }^{41}$ Therefore, evaluation of the effects of FAs on activated fibroblasts by cytokines, such as TGF- $\beta 1$ and IL- $1 \beta$, released from these cells is required to elucidate the effects on keloid tissues. Similarly, evaluation of the butyrate/DHA combination in an in vivo study is also required; however, a human model of keloid has not been established yet.

\section{CONCLUSIONS}

We demonstrated the antifibrogenic effects of butyrate and DHA on KFBs. These findings could contribute to the development of novel therapy for dermal fibroproliferative disorders. $\square$ 


\section{REFERENCES}

1. Seifert 0, Mrowietz U. Keloid scarring: bench and bedside. Arch Dermatol Res. 2009;301:259-72.

2. Poochareon VN, Berman B. New therapies for the management of keloids. J Craniofac Surg. 2003;14:654-7.

3. Ragoowansi R, Cornes PG, Moss AL, Glees JP.Treatment of keloids by surgical excision and immediate postoperative single-fraction radiotherapy. Plast Reconstr Surg. 2003:111:1853-9.

4. Shih B, Bayat A. Genetics of keloid scarring. Arch Dermatol Res. 2010;302:319-39.

5. Kang JS, Liu C, Derynck R. New regulatory mechanisms of TGF-beta receptor function. Trends Cell Biol. 2009;19:385-94.

6. Sarrazy V, Billet $F$, Micallef $L$, Coulomb B, Desmoulière A. Mechanisms of pathological scarring: role of myofibroblasts and current developments. Wound Repair Regen. 2011;19:s10-5.

7. Tomasek JJ, Gabbiani G, Hinz B, Chaponnier C, Brown RA. Myofibroblasts and mechano-regulation of connective tissue remodeling. Nat Rev Mol Cell Biol. 2002;3:349-63

8. Yeh FL, Shen HD, Lin MW, Chang CY, Tai HY, Huang MH. Keloid-derived fibroblasts have a diminished capacity to produce prostaglandin E2. Burns. 2006;32:299-304

9. Hayashi T, Nishihira J, Koyama Y, Sasaki S, Yamamoto Y. Decreased prostaglandin E2 production by inflammatory cytokine and lower expression of EP2 receptor result in increased collagen synthesis in keloid fibroblasts. J Invest Dermatol. 2006;126:990-7.

10. Davie JR. Inhibition of histone deacetylase activity by butyrate. J Nutr. 2003;133:2485S-2493S

11. Roy CC, Kien CL, Bouthillier L, Levy E. Short-chain fatty acids: ready for prime time? Nutr Clin Pract. 2006;21:351-66.

12. Usami M, Kishimoto K, Ohata A, Miyoshi M, Aoyama M, Fueda Y, et al. Butyrate and trichostatin $A$ attenuate nuclear factor kappaB activation and tumor necrosis factor alpha secretion and increase prostaglandin E2 secretion in human peripheral blood mononuclear cells. Nutr Res. 2008;28:321-8.

13. Bülow R, Fitzner B, Sparmann G, Emmrich J, Liebe S, Jaster R. Antifibrogenic effects of histone deacetylase inhibitors on pancreatic stellate cells. Biochem Pharmacol. 2007;74:1747-57.

14. Niki T, Rombouts K, De Bleser P, De Smet K, Rogiers V, Schuppan D, et al. A histone deacetylase inhibitor, trichostatin $A$, suppresses myofibroblastic differentiation of rat hepatic stellate cells in primary culture. Hepatology. 1999;29:858-67.

15. Rishikof DC, Ricupero DA, Liu H, Goldstein RH. Phenylbutyrate decreases type I collagen production in human lung fibroblasts. J Cell Biochem. 2004:91:740-8.

16. Torii K, Maeshige N, Aoyama M, Imai M, Tabuchi H, Miyoshi M et al. Antifibrogenic effects of short chain fatty acids and $\omega-3$ polyunsaturated fatty acids on human dermal fibroblasts. 4th Congress of the World Union of Wound Healing Societies. 2012.

17. Im DS. Omega-3 fatty acids in anti-inflammation (pro-resolution) and GPCRs. Prog Lipid Res. 2012;51:232-7.

18. Siddiqui RA, Harvey KA, Xu Z, Bammerlin EM, Walker C, Altenburg JD. Docosahexaenoic acid: a natural powerful adjuvant that improves efficacy for anticancer treatment with no adverse effects. Biofactors. 2011;37:399-412.

19. Victory R, Saed GM, Diamond MP. Antiadhesion effects of docosahexaenoic acid on normal human peritoneal and adhesion fibroblasts. Fertil Steril. 2007;88:1657-62.

20. Bianchini F, Giannoni E, Serni S, Chiarugi P, Calorini L. 22 : 6n-3 DHA inhibits differentiation of prostate fibroblasts into myofibroblasts and tumorigenesis. $\mathrm{Br} J$ Nutr. 2012;108:2129-37.

21. Yamamoto K, Itoh T, Abe D, Shimizu M, Kanda T, Koyama T, et al. Identification of putative metabolites of docosahexaenoic acid as potent PPAR $\gamma$ agonists and antidiabetic agents. Bioorg Med Chem Lett. 2005;15:517-22.

22. Ghosh AK, Wei J, Wu M, Varga J. Constitutive Smad signaling and Smaddependent collagen gene expression in mouse embryonic fibroblasts lacking peroxisome proliferator-activated receptor-gamma. Biochem Biophys Res Commun. 2008;374:231-6.

23. Sarr D, Aldebert D, Marrama L, Frealle E, Gaye A, Brahim HO, et al. Chronic infection during placental malaria is associated with up-regulation of cycloxygenase-2. Malar J. 2010:9:45

24. Liao CJ, Huang YH, Au HK, Wang LM, Chu ST. The cancer marker neutrophil gelatinase-associated lipocalin is highly expressed in human endometrial hyperplasia. Mol Biol Rep. 2012;39:1029-36.

25. Meng W, Xia Q, Wu L, Chen S, He X, Zhang L et al. Downregulation of TGF-beta receptor types II and III in oral squamous cell carcinoma and oral carcinomaassociated fibroblasts. BMC Cancer. 2011;11:88.
26. Heinemeier KM, Bjerrum SS, Schjerling P, Kjaer M. Expression of extracellular matrix components and related growth factors in human tendon and muscle after acute exercise. Scand J Med Sci Sports. 2013;23:e150-61.

27. Aoyama M, Kotani J, Usami M. Butyrate and propionate induced activated or nonactivated neutrophil apoptosis via HDAC inhibitor activity but without activating GPR-41/GPR-43 pathways. Nutrition. 2010;26:653-61.

28. Dzierzewicz Z, Szczerba J, Lodowska J, Wolny D, Gruchlik A, Orchel A, et al. The role of Desulfovibrio desulfuricans lipopolysaccharides in modulation of periodontal inflammation through stimulation of human gingival fibroblasts. Arch Oral Biol. 2010;55:515-22.

29. Mohana Kumar B, Song HJ, Cho SK, Balasubramanian S, Choe SY, Rho GJ. Effect of histone acetylation modification with sodium butyrate, a histone deacetylase inhibitor, on cell cycle, apoptosis, ploidy and gene expression in porcine fetal fibroblasts. J Reprod Dev. 2007;53:903-13.

30. Abramova MV, Pospelova TV, Nikulenkov FP, Hollander CM, Fornace AJ Jr, Pospelov VA.. G1/S arrest induced by histone deacetylase inhibitor sodium butyrate in E1A + Ras-transformed cells is mediated through down-regulation of E2F activity and stabilization of beta-catenin. J Biol Chem. 2006;281:21040-51.

31. Jafarpour F, Hosseini SM, Hajian M, Forouzanfar M, Ostadhosseini S, Abedi P, et al. Somatic cell-induced hyperacetylation, but not hypomethylation, positively and reversibly affects the efficiency of in vitro cloned blastocyst production in cattle. Cell Reprogram. 2011;13:483-93.

32. Skender B, Vaculova AH, Hofmanova J. Docosahexaenoic fatty acid (DHA) in the regulation of colon cell growth and cell death: a review. Biomed Pap Med Fac Univ Palacky Olomouc Czech Repub. 2012;156:186-99.

33. Fan YY, Zhang J, Barhoumi R, Burghardt RC, Turner ND, Lupton JR, et al. Antagonism of CD95 signaling blocks butyrate induction of apoptosis in young adult mouse colonic cells. Am J Physiol. 1999;277:C310-9.

34. Tang YA, Wen WL, Chang JW, Wei TT, Tan YH, Salunke S, et al. A novel histone deacetylase inhibitor exhibits antitumor activity via apoptosis induction, F-actin disruption and gene acetylation in lung cancer. PLoS One. 2010;5:e12417.

35. Sandbo N, Lau A, Kach J, Ngam C, Yau D, Dulin NO. Delayed stress fiber formation mediates pulmonary myofibroblast differentiation in response to TGF- $\beta$. Am J Physiol Lung Cell Mol Physiol. 2011;301:L656-66.

36. Ghosh AK, Bhattacharyya S, Lakos G, Chen SJ, Mori Y, Varga J. Disruption of transforming growth factor beta signaling and profibrotic responses in normal skin fibroblasts by peroxisome proliferator-activated receptor gamma. Arthritis Rheum. 2004:50:1305-18

37. Taniura S, Kamitani $\mathrm{H}$, Watanabe T, Eling TE. Transcriptional regulation of cyclooxygenase-1 by histone deacetylase inhibitors in normal human astrocyte cells. J Biol Chem. 2002;277:16823-30

38. Sandulache VC, Parekh A, Li-Korotky H, Dohar JE, Hebda PA. Prostaglandin E2 inhibition of keloid fibroblast migration, contraction, and transforming growth factor (TGF)-beta1-induced collagen synthesis. Wound Repair Regen. 2007;15:122-33

39. Bozyk PD, Moore BB. Prostaglandin E2 and the pathogenesis of pulmonary fibrosis. Am J Respir Cell Mol Biol. 2011;45:445-52.

40. Messadi DV, Doung HS, Zhang Q, Kelly AP, Tuan TL, Reichenberger E, et al. Activation of NFkappaB signal pathways in keloid fibroblasts. Arch Dermatol Res. 2004:296:125-33.

41. Mahdavian Delavary B, van der Veer WM, van Egmond M, Niessen FB, Beelen RH. Macrophages in skin injury and repair. Immunobiology. 2011;216:753-62.

How to cite this article: Torii K, Maeshige N, Aoyama-Ishikawa M, Miyoshi M, Terashi H, Usami M. Combination therapy with butyrate and docosahexaenoic acid for keloid fibrogenesis: an in vitro study. An Bras Dermatol. 2017;92(2):184-90. 